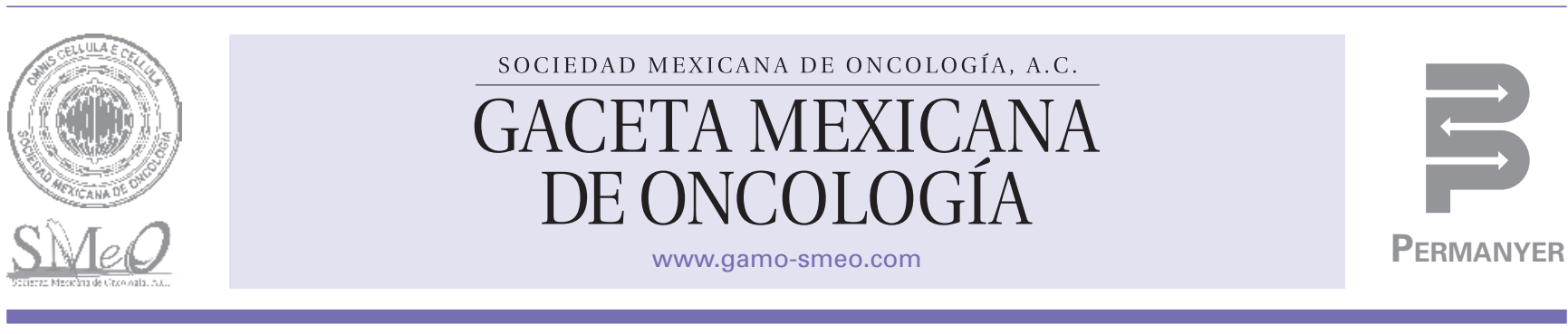

\title{
EDITORIAL
}

\section{Disciplina, hábitos y cáncer}

\author{
Joel Mario Salazar-Cavazos
}

Opción Oncología, Monterrey, N.L., México

Recibido el 31 de octubre de 2017; aceptado el 1 de noviembre de 2017

Disponible en Internet el 4 de diciembre de 2017

El estilo de vida puede impactar en forma directa y contundente sobre las probabilidades de que un individuo llegue a padecer cáncer en el transcurso de su existencia. Esta influencia puede ser positiva o negativa, según sea el caso, y está directamente relacionada con la disciplina en lo que respecta a las costumbres alimenticias y a la actividad física que realice regularmente. Es bien sabido que el sobrepeso, la obesidad, el tabaquismo, la ingesta de alcohol y el sedentarismo son algunos de los factores ambientales que podemos modificar para reducir el riesgo de padecer algunas enfermedades oncológicas. De antemano aclaro que excluyo de esta lista a las personas que sufren un aumento de peso secundario a enfermedades metabólicas, endocrinas o a alguna otra enfermedad menos común.

El cáncer es un problema de salud global, y México no es la excepción, pues constituye la tercera causa de muerte después de las enfermedades cardiovasculares y la diabetes mellitus con sus complicaciones (por cierto, en ambos casos influyen de manera evidente los mismos factores que en muchos tipos de cáncer). En lo que concierne a la incidencia, los cánceres más frecuentes son el de próstata, el colorrectal y el de pulmón para los varones, mientras que en el género femenino encabezan la lista el cáncer de mama, el de pulmón y el colorrectal. Por otro lado, los tipos de neoplasias malignas que provocan la mayor cantidad de muertes entre los hombres son la de pulmón, la de próstata y la colorrectal; en el caso de las damas, la de pulmón, la de mama y la colorrectal son las de mayor mortalidad'.

El sobrepeso y la obesidad se han convertido en una pandemia, y en los últimos años se ha incrementado la inciden- cia de ambas entidades, aun en países donde tradicionalmente esto no constituía un problema de salud pública. Se considera que los cambios en el estilo de vida y la globalización de las grandes y exitosas cadenas de comida rápida han jugado un importante papel en la génesis de este serio problema. Es mucho más fácil adoptar los malos hábitos alimenticios de los países con alto índice de sobrepeso que la dieta sana y balanceada que aún predomina en pocos países del mundo o, mejor dicho, en pocas y pequeñas regiones del mundo. Nos escondemos detrás de la excusa «no tengo tiem po» para acceder cotidianamente a los centros de comida chatarra que abundan en la actualidad.

En nuestro país, el $70 \%$ de la población sufre sobrepeso (índice de masa corporal [IMC] > 25) y el $36 \%$, obesidad $(I M C>30)$. Éstas se han ligado con mucha evidencia a diferentes tipos de cáncer, entre los que destacan los de mama, páncreas, riñón, endometrio, esófago, colon y recto, por mencionar algunos. En EE.UU. se estiman 100,000 casos nuevos de cáncer por año que podrían estar relacionados con los hábitos dietéticos.

Entre 1990 y 2015, se analizaron datos de 195 países al: rededor del mundo. Para el año 2015, había 107.7 millones de niños y 603.7 millones de adultos obesos, lo que significa que en los últimos 15 años la prevalencia de esta enfer-y medad se ha duplicado en más de 70 de estos países y se ha incrementado en la mayoría del resto. Se calcula que ele sobrepeso/obesidad causó cuatro millones de muertes. El crecimiento en la incidencia de obesidad es muy evidente a partir de los 15 años de edad, a diferencia de lo que ocurrian en las generaciones anteriores, en las que el aumento de

*E-mail para correspondencia: salazar@opciononcologia.com (J.M. Salazar-Cavazos) 
peso llegaba en una época más tardía de la vida. El porcentaje de obesidad en adultos fue a la alza constante desde el inicio hasta el final del estudio².

En un análisis realizado en 2009 en población urbana americana, llama en exceso la atención que, a diferencia de la alta conciencia que se tiene de la relación del tabaquismo, la exposición solar y la genética con el riesgo de cáncer (más del $90 \%$ de las personas lo sabían o al menos lo habían escuchado), sólo alrededor del $50 \%$ de los encuestados habían oído de la asociación entre obesidad y cáncer. La misma cifra o menor incluso conocía el riesgo incrementado de padecer cáncer con una dieta baja en vegetales, con la ingesta de alcohol, con el alto consumo de carnes rojas y con una pobre actividad física. Cuesta trabajo creer que en estos tiempos, cuando la mayoría de la población tiene acceso a información amplia y de primera mano, no se asocie la obesidad con el cáncer. Es posible que los programas de salud pública no lleguen a la mitad de la gente o puede que simplemente se ignoren; me inclino a pensar en lo segundo, y con mayor razón insisto en que el cáncer puede ser provocado por nuestra indisciplina en la vida cotidiana. Éste es sin duda uno de los motivos por los que en ese país aumentó el número de personas con obesidad de 35 millones en 2001 a 51 millones en el año del estudio, 2009. Es preciso que las autoridades de salud en México conozcan estos mismos datos en nuestra población (probablemente sean muy similares), para así enfocar correctamente los esfuerzos que se hacen para educar y concientizar sobre la gravedad y consecuencias de este problema.

En otro estudio, al cuestionar sobre las principales causas de muerte, más del 35\% de los entrevistados mencionaron el cáncer como la número uno, incluso por encima de las enfermedades cardiovasculares, la diabetes y la enfermedad vascular cerebral. Curiosamente al preguntar a este mismo grupo de individuos si estas cuatro causales de muerte podían ser prevenidas, sólo el $20 \%$ consideró que el cáncer podría serlo; en cambio, las otras tres entidades fueron consideradas entre el 30 y casi el $50 \%$ de los casos. Este es otro dato que llama mucho la atención, y de nuevo pongo sobre la mesa si los programas de salud pública en materia de prevención del cáncer están siendo bien enfocados o si las autoridades sanitarias están luchando contra la apatía y el desinterés de la población, destinando recursos que están rindiendo menos frutos de los esperados.

Si consideramos el impacto que pudiéramos causar entre algunos de los diferentes factores de riesgo que se han descrito para desarrollar cáncer, nos podríamos encontrar con que la obesidad se encuentra en vías de suplir al tabaquismo como la principal causa prevenible de cáncer. El porcentaje de casos de cáncer atribuidos a la obesidad es muy variable, y puede llegar a ser tan alto como del $40 \%$ en algunos tipos como los de endometrio y esófago (adenocarcinoma). Así mismo, el sobrepeso y la obesidad contribuyen a una de cada cinco muertes relacionadas con el cáncer. Por lo anterior podemos concluir que la obesidad es el nuevo tabaquismo en lo que respecta a riesgo de contraer cáncer.

El sobrepeso y la obesidad pueden causar cáncer por diferentes mecanismos: hipoxia celular, el tejido graso produce más altos niveles de estrógeno en la población femenina, también se pueden presentar niveles incrementados de insulina con resistencia a la misma, en las personas obesas se puede producir un estado crónico o subagudo de inflamación y finalmente los adipocitos pueden tener efectos reguladores en el crecimiento de ciertos tumores.

Con la obesidad, no sólo se tiene un mayor riesgo de padecer algunos tipos de cáncer, sino que en las personas que ya lo tienen se puede presentar con peor pronóstico y hay mayor tasa de recurrencia, aumento de metástasis, menor supervivencia, más alta mortalidad y segundos cánceres $^{3}$.

Se ha demostrado que un aumento en el IMC impacta en una mayor incidencia de muchas neoplasias malignas; entre las más documentadas en hombres se encuentran el adenocarcinoma de esófago, tiroides, colorrectal, páncreas, próstata, riñón e hígado, mientras que en las mujeres son los de endometrio, vesícula biliar, adenocarcinoma de esófago, riñón, mama (posmenopausia), colorrectal y páncreas ${ }^{4}$.

Un IMC elevado (> 25) puede causar la muerte por diferentes motivos, y los más frecuentes son: isquemia cardíaca; enfermedad vascular cerebral, otras enfermedades vasculares, diabetes mellitus, diversos tipos de cáncer y enfermedades renales y hepáticas no neoplásicas, entre otras ${ }^{5}$.

La asociación entre algunas enfermedades metabólicas relacionadas con el sobrepeso y la obesidad como la diabetes mellitus también impacta aumentando el riesgo de ciertos tipos de cáncer como el de mama, endometrio e hígado. Así mismo, se puede incrementar la mortalidad, ya que esta coexistencia ha demostrado ser un factor de mal pronóstico en ciertas neoplasias malignas como el cáncer de mama y el colorrectal $^{6,7}$. Por el contrario, existe información de que en este subgrupo de pacientes la metformina puede disminuir el riesgo de cáncer y su mortalidad, disminuyendo las me tástasis distantes, y probablemente mejore los resultados de la quimioterapia en el cáncer de mama.

La actividad física regular, especialmente si se inicia desde edades tempranas, tiene como resultado una disminución de hasta el $20 \%$ en el riesgo de desarrollar cáncer de mama y colorrectal. Las sociedades modernas son más sedentarias, y se considera que el $5 \%$ de los cánceres puede estar asociado directamente con poca práctica de ejercicio. Además, la actividad aeróbica regular puede traer diferentes beneficios en pacientes diagnosticados con cáncer: disminuye algunos efectos adversos del tratamiento, mejora la calidad de vida, reduce el estrés emocional, hay menor fatiga, aumenta la capacidad funcional y fortalece el estado físico, disminuye el sobrepeso, reduce la resistencia a la insulina y los niveles de glucosa sérica, y además baja los niveles de estrógeno y/o andrógenos. Las horas de ejercicio por semana están directamente relacionadas con la sobrevida y la mortalidad en pacientes con diagnóstico de cáncer de mama, y el grupo de mejores resultados es el que practica actividades físicas más de $9 \mathrm{~h}$ por semana ${ }^{8,9}$.

También se han estudiado las intervenciones quirúrgicas enfocadas al control del sobrepeso y obesidad, y se asocian a una disminución de los tumores relacionados con la obesidad, así como a una reducción de la mortalidad por cáncer ${ }^{10}$.

La pregunta más difícil de contestar es si tratar la obesidad es más complejo que tratar el cáncer. ¿Qué porcentaje de personas que padecen obesidad logran erradicarla? ¿Es probable que la tasa de curación de muchos tipos de cáncer sea más alta que la de la obesidad? ¿Cuáles son los factores que lo hacen tan complejo? ¿Hay mediadores físicos que lo 
dificulten? ¿Puede considerarse la obesidad una enfermedad emocional? ¿Es un poco de todo lo anterior o simplemente en las últimas décadas hemos cambiado los satisfactores personales y sentimos la obligación de cubrir cada día nuestras «necesidades» inmediatas, sin pensar en las consecuencias a mediano o largo plazo? ¿Somos capaces de cambiar nuestra salud por el placer de comer o beber lo que se nos antoje sin importar lo que pasará mañana? ¿Por qué, si sabemos el impacto negativo tan alto que puede llegar a tener, no somos capaces de controlarla y/o resolverla? En países como México y EE.UU., que ocupan el primer y el segundo lugar mundial de obesidad, más del $90 \%$ de los ciudadanos no cumplen con los requerimientos en el consumo de granos, frutas y vegetales. ¿Por qué como sociedad seguimos tomando alcohol, fumando cigarrillos, siendo sedentarios y manteniendo un IMC por encima de lo recomendado? ¿Con qué derecho el profesional de la salud que conoce los riesgos le puede exigir a su paciente, que «no sabe», que deje ciertas prácticas en su vida cotidiana que son deletéreas para su salud? En fin, creo que dejo más dudas que respuestas, y, la verdad, lo hago intencionalmente, pues es un tema para el autoanálisis. ¿Qué tanto tendríamos que cambiar para ayudar más a nuestros pacientes... y a nosotros mismos?

\section{BIBLIOGRAFÍA}

1. Siegel R, Ward E, Brawley O, Jemal A. Cancer statistics, 2011: the impact of eliminating socioeconomic and racial disparities on premature cancer deaths. CA Cancer J Clin. 2011;61(4):212-36.

2. GBD 2015 Obesity Collaborators, Afshin A, Forouzanfar MH, et al. Health Effects of Overweight and Obesity in 195 Countries over 25 Years. N Engl J Med. 2017;377(1):13-27.

3. Ewertz M, Jensen MB, Gunnarsdóttir KÁ, et al. Effect of obesity on prognosis after early-stage breast cancer. J Clin Oncol. 2011;29(1):25-31.

4. Renehan AG, Tyson M, Egger M, Heller RF, Zwahlen M. Body-mass index and incidence of cancer: a systematic review and meta-analysis of prospective observational studies. Lancet. 2008;371(9612):569-78.

5. Prospective Studies Collaboration, Whitlock G, Lewington S, et al? Body-mass index and cause-specific mortality in 900000 adults: collaborative analyses of 57 prospective studies. Lancet. 2009; 373(9669):1083-96.

6. Colorectal Dis. 2011.

7. Duggan C, Irwin ML, Xiao L, et al. Associations of insulin resistance and adiponectin with mortality in women with breast cancer. $\mathrm{J}$ Clin Oncol. 2011;29(1):32-9.

8. Holmes, et al. JAMA. 2005.

9. Irwin ML, Smith AW, McTiernan A, et al. Influence of pre- and postdiagnosis physical activity on mortality in breast cancer survivors: the health, eating, activity, and lifestyle study. J Clin Oncol. 2008; 26(24):3958-64.

10. Ashrafian H, Ahmed K, Rowland SP, et al. Metabolic surgery and cancer? protective effects of bariatric procedures. Cancer. 2011;117(9):1788-99. 